\title{
29. NUMERICAL MODELING OF SEDIMENT DEFORMATION LINKED TO SUBDUCTION: MECHANICAL MODEL AND COMPARISON WITH BARBADOS RIDGE COMPLEX ${ }^{1}$
}

\author{
Kombe Ngokwey, Direction de Recherche Géologie-Géochimie, Institut Français du Pétrole²
}

\begin{abstract}
A two dimensional finite element analysis is used to describe the mechanical behavior of a sedimentary cover subjected to compressive forces resulting from subduction. My approach consists of the construction, for the crust-sediment set, of mechanical models for a given rheology, and the study of the deformation in relation to boundary conditions. I assume that rocks behave like viscoelastic bodies during the process of geological deformation.

Consideration has been given to the following parameters: the presence of a décollement with acoustically stratified sediments beneath, the lithology (composition of nature) and thickness of sediments above the décollement, and the convergence rate. This enables me to compare observations made in the specific case of the Lesser Antilles arc.

The main results are (1) the sedimentary covery displays a compressional stress field; (2) a décollement has appreciable effects on the mechanism of low-angle thrust faulting; (3) variations in sediment strength produce variations in strain rates and thus in the ability of sedimentary cover to be deformed; and (4) sediment thickness and the convergence rate affect the amplitude of deformation.
\end{abstract}

\section{INTRODUCTION}

Plate tectonic theory holds that the lithosphere can be divided into a small number of rigid plates interacting only on their boundaries, along which major tectonic and seismic activity occurs. Subduction zones are the consuming boundaries where plates of oceanic lithosphere sink into the mantle and are overridden by other plates, which may be oceanic (island arc) or continental (active continental margin) in nature. A major emphasis of current work in geodynamics is the structural and the sedimentological study of such active margins. Our investigation is restricted, from a structural viewpoint, to the uppermost part of the lithosphere.

Sediment behavior in the subduction process has been discussed by several authors (Oxburgh and Turcotte, 1971; Seely et al., 1974; Mascle et al., 1976). In general, a portion of the sediments carried by the subducted plate are very deformed and build up into an accretionary prism. Observations at active margins have shown that, depending upon the degree of deformation, such prisms acquire various morphologies and internal structures. Subduction is still the principal driving force, but by itself it could create neither the diversity nor extent of those structures. Consideration is thus given to other parameters such as the lithology and thickness of sediments involved, the convergence rate, and the presence of a décollement. In order to investigate the contribution of each of those parameters to deformation, an evaluation based on solid mechanics and rock mechanics can be made by using the finite element method. This approach consists of the construction, for the ocean crust-sediment system, of mechanical models that

\footnotetext{
${ }^{1}$ Biju-Duval, B., Moore, J. C., et al., Init. Repts. DSDP, 78A: Washington (U.S. Govt. Printing Office).

2 Address: Direction de Recherche Géologie-Géochimie, Institut Français du Pétrole, 92506 Rueil-Malmaison Cedex, France.
}

are based upon a given rheology. The deformation can then be studied in terms of various assigned boundary conditions. Models are derived with the assumption that rocks behave like viscoelastic bodies during the process of geological deformation. I present such a study here, for comparison to the Barbados Ridge, an accretionary complex east of the Lesser Antilles arc. The study is qualitative and is designed to show whether the hypotheses adopted permit us to generate models that approximate the observed styles of deformation.

\section{NUMERICAL MODELING}

\section{Rheology-Experimental Basis}

Mandel (1966) gives experimental results that provide guidelines for the behavior of the solid bodies. A bar of steel subjected to tensile force $F$ (Fig. 1A) displays a strain $\epsilon$, which develops at a rate $\dot{\epsilon}$, which develops at a rate $\dot{\epsilon}=d \epsilon / d t$. Assuming that experiments are carried out at $\dot{\epsilon}=$ constant, one may observe that Figure 1B shows a reversible part $A B$ and a part (after B) characterized by permanent large deformations leading to failure: the permanent deformations characterize the plasticity of the material. Figure 1C illustrates a test done for different values of $\dot{\epsilon}$. For the same $\epsilon, F$ is an increasing function of $\dot{\epsilon}$ : the faster the deformation is, the more the material seems rigid. The fact that $F$ depends upon $\dot{\epsilon}$ (and thus upon the time) characterizes the viscosity of the material. The reversible initial phase is the definition of elasticity. Thus real deformation is elastic-viscous-plastic, with elasticity or viscoelasticity, or viscoplasticity dominant. For subduction zones, Seely et al. (1974) have proposed a qualitative geometric interpretation of sedimentary accretion. The sedimentary cover is subjected to oblique shear. Pautot (1975) suggests that the sedimentary column reacts as a solid body when subjected to shear and as a viscous body to allow creep. Thus it seems that rock rheology and the loading to 

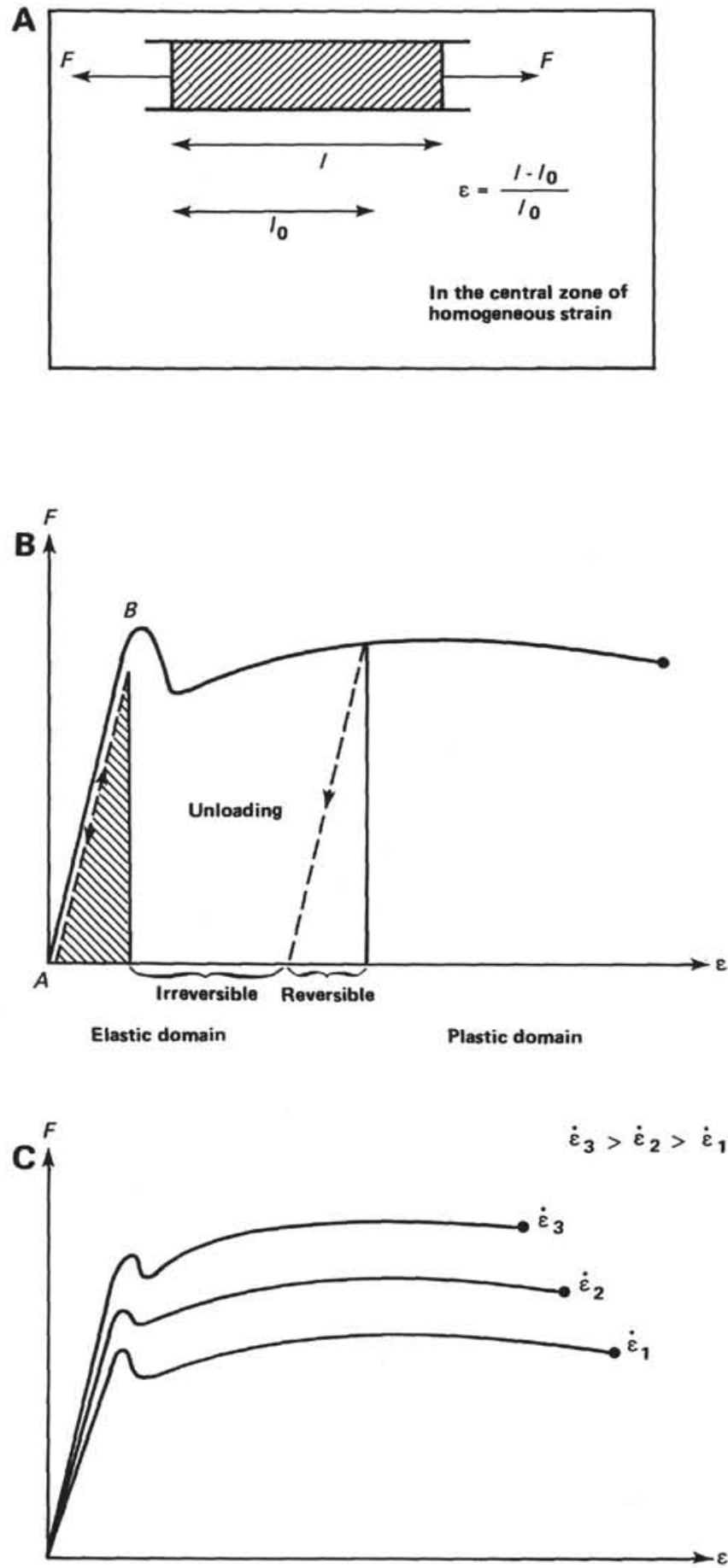

Figure 1. Rheology-experimental basis. A and B. Elasticity and plasticity. C. Viscosity.

which rocks are subjected are such that is essential to take into account, in addition to instantaneous deformation, the deformation and relaxation phenomena associated with viscosity. This is the reason why we have chosen to study viscoelastic behavior.

A viscoelastic body is a medium in which strains depend both on stresses and stress history:

$$
\sigma(t)=D\left|\epsilon_{\mathrm{tot}}(t)-\epsilon_{\nu}(t)\right|
$$

with $D=$ elasticity, matrix, $\epsilon_{\mathrm{tot}}=$ total strain, and $\epsilon_{\mathrm{v}}=$ viscous strain. This type of body is mechanically equivalent to an assemblage having a series of elastic and of viscoelastic elements (or Kelvin-Voigt elements) (Fig. 2). Equations for this rheological scheme may be written as:

$$
\begin{aligned}
\sigma & =D \epsilon_{e} \\
\sigma & =D_{v}\left|\frac{a}{b} \epsilon_{v}+\frac{1}{a} \frac{\mathrm{d} \epsilon_{v}}{d t}\right| \\
\epsilon_{\text {tot }} & =\epsilon_{e}+\epsilon_{v}
\end{aligned}
$$

with $a=1 / \eta$ ( $\eta=$ viscosity), $b=E_{v} / \eta$ ( $E_{v}=$ Young's Modulus of Kelvin-Voigt elements), $D_{v}=$ viscosity matrix, and $\epsilon_{e}=$ elastic strain.

Our purpose is the calculation, at every moment, of displacement and stress. Time steps $\Delta t$ are used, with the assumptions that the applied loads are constant during the time and that parameters such as mechanical properties and so on are also constant over the interval $|t-\Delta t, t|$ considered. Thus integration of Equation 3 gives:

$$
\epsilon_{v}(t)=\epsilon_{v}(t-\Delta t) e^{-b \Delta t}+\frac{a}{b} D_{v}^{-1} \sigma(t-\Delta t)\left(1-e^{-b \Delta t}\right)
$$

Then equations 2,4 , and 5 can be resolved by finite element procedures.

\section{Sediment Deformation-Parameter Sensitivities}

The state of sediment deformation can be represented as a function of a series of parameters. These are: the presence of a décollement, the convergence rate, and the lithology and thickness of the sediment involved. These parameters are reviewed in order to investigate their sensitivities in the sediment deformation process. Our study is a qualitative one, and the models described here give us information only about the likelihood that a given phenomenon will occur.

\section{Mechanical Models-Uncertainties Linked to Plate-Tectonic and Geological Problems}

When the convergence rate of two lithospheric plates is estimated to be around $2 \mathrm{~cm} / \mathrm{yr}$., this means that their

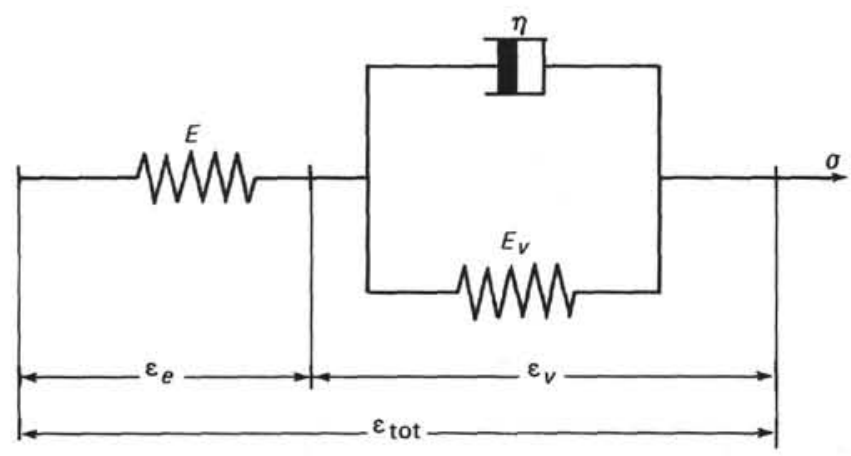

Figure 2. Viscoelasticity-rheological scheme. $(E=$ Young's Modulus of elastic elements, $E_{v}=$ Young's Modulus of viscoelastic elements, and $v=$ viscosity.) 
convergence is assumed to be $20 \mathrm{~km}$ during $1,000,000$ yr. But nobody can insure that this total convergence of $20 \mathrm{~km}$ has not been acquired in a discontinuous way, with, for instance, $201 \mathrm{~cm} / \mathrm{yr}$. during $0.5 \%$ of the total duration and $1 \mathrm{~cm} / \mathrm{yr}$. during $99.5 \%$ of this duration, or $38.1 \mathrm{~cm} / \mathrm{yr}$. during $5 \%$ of the duration and $0.1 \mathrm{~cm} / \mathrm{yr}$. during $95 \%$ of this duration. It is obvious that when considering the deformations, whose strain-rate scale varies from 1 to 100 or even from 1 to 1000 , the mechanisms involved will not be the same. Schematically, let us say that high and low strain rates will favor elasticplastic and viscous phenomena, respectively.

In addition, large rocks groups are modeled and, in each of the great subdivisions of the model, are supposed to be continuous and homogeneous. In reality, these groups are not at any time of their history continuous or homogeneous. It is difficult to know which mechanical parameters may be assigned to an imaginary continuous medium in order to confer on it the natural behavior of the actual rocks. Thus no conclusions can be drawn when mechanical tests have not been done; the values of the coefficients measured on a rock sample give very few indications of the behaviors of the rock at the scale of an outcrop or larger.

\section{Consequences of These Uncertainties}

Between elastic moduli $E_{r}$ and $E_{v r}$ (see Fig. 2) and those used for calculations $E_{c}$ and $E_{v c}$, there is a factor of proportionality $k$, which is unknown:

$$
\begin{aligned}
E_{c} & =k E_{r} \\
E_{v c} & =k E_{v r}
\end{aligned}
$$

This means that the ratio $\alpha=E_{r} / E_{v r}$ is unknown too.

There is, besides these uncertainties, an uncertainty concerning condition assigned to the model (schematized on Fig. 2) by the value of the stress $\sigma$. If we assume that this stress is known to be a little less than the multiplicative factor, one may observe that in Equation 1, this is equivalent to applying again an unknown multiplicative factor to the elastic parameters. This uncertainty in the assumptions concerning stresses at the boundaries of the subducted plate does not induce new uncertainties with respect to the uncertainties in the mechanical properties.

The discussion of uncertainties in mechanical models indicates we cannot know if the significant period for stress accumulation is $10^{6}$ or $10^{3}$ yr. In Equation 5, one may find out that the time is adimensional in the iterative process, because $b$ has the dimension $|\mathrm{T}|^{-1}$, and $a /$ $b$ is homogeneous with respect to pressure. Thus the generality of the calculations is not restricted when $\eta=$ $E_{v}$ and the time relaxation $1 / b$ equals 1 . Then, the calculations can be made with the time steps $\Delta t$ covering a prescribed total period $t=10$, which enables us to be certain that the asymptotic state is reached at the end of the calculations. In other words, time is normalized.

\section{Significance of Stresses Computed and Associated with These Uncertainties}

The total stresses computed are the sum of two tensors:

$$
|\sigma|=|S|+k|T|
$$

where $|\sigma|=$ total stresses, $|S|=$ "static stresses" (resulting from gravity and interstitial fluid pressure, which is neglected here); and $k|T|=$ "tectonic stresses" associated with nonstatic boundary conditions assigned to the model; $k$ means that these stresses are known to be a little less than integral factors, which are unknown.

In practice, we have choosen $k$ large enough so that the term $k|T|$ is predominant. Thus the variations of tectonic stresses are more evident in the models. It must be understood that the values of stresses have no physical meaning when they are considered in isolation. This is, for example, the case when the calculations produce stresses of several kilobars. Such quantities are meaningful only when they are compared between models.

Let us examine this system using a simple example. Suppose that at a given point $\mathrm{A}$, the total horizontal compression is 16,000 bars, whereas the horizontal component of geostatic stress at the same point is 1000 bars. At another point B, the total horizontal compression is assumed to be 2000 bars in magnitude and the horizontal component of geostatic stress, 500 bars. This means that at point $\mathrm{A}$, the horizontal tectonic stress is ten times greater than at point $\mathrm{B}$, because $(16,000-1000) /$ $(2000-500)=10$.

Such a conclusion is obviously dependent on the choice of points examined and the stresses acting at each point. The stress differences can only be evaluated by considering two or more points, not points in isolation. This is true whatever the unknown factor $k$ may be.

\section{Finite Element Grids}

In our finite element analysis, we use a quadrilateral isoparametric element with eight nodes. The finite element grid representing the general features of a typical subduction zone is shown in Figure 3. The total numbers of nodes and elements assigned here are 458 and 123 , respectively. Our model space is composed of four mechanical units. The first one corresponds to the overriding plate, the second to the subducted plate, and the third represents the transitional layer along which relative motion occurs between units. The fourth unit corresponds to the unsubducted sedimentary cover. The model is $400 \mathrm{~km}$ long and subducted plate has been taken as being $7 \mathrm{~km}$ thick.

The sediments are stratified material. Such a material is considered to be orthotropic and is characterized by five independent elastic constants: $E_{1}, E_{2}, G_{2}, v_{1}, v_{2} . E_{1}$, $v_{1}\left(G_{1}\right.$ is dependent) are associated with the behavior in the stratification plane, and $E_{2}, G_{2}, v_{2}$ with a direction normal to this. Nevertheless, for isotropic material $\left(E_{1}\right.$ $=E_{2}$ and $\left.v_{1}=v_{2}\right), G_{2}$ is given by

$$
G_{2}=E_{1} / 2\left(1+\nu_{1}\right)
$$



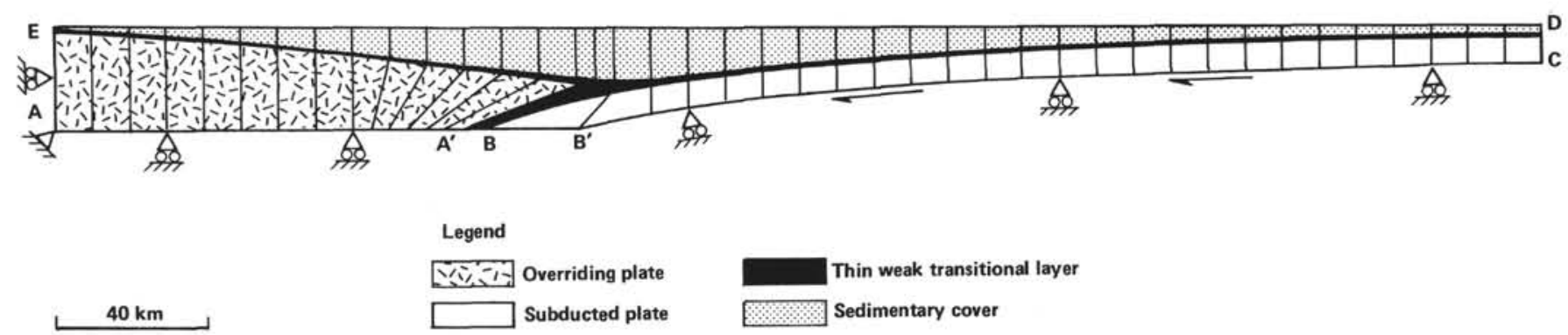

Figure 3. Finite element net and boundary conditions used. Mechanical units are shown. (See the text on boundary conditions for an explanation of $\mathrm{AE}, \mathrm{CD}, \mathrm{BC}$, and so on.)

The previous discussion on the physical heterogeneity of sediments indicates that it is reasonable to reduce the constitutive media of the model to some general classes: "soft," "rigid," "very rigid" material, and so on. If the rigidities of the media are preserved, each with respect to the others, such a description will lead to an accurate scheme of deformation. The mechanical properties used are tabulated in Table 1.

We consider four different cases (Figs. 4-6). The first case investigates the contact effect between sediments and both overriding and subducted plates in the presence (DEC2) or absence (DEC1) of a thin weak transitional layer. The second case (NA1 and NA2) corresponds to the study of the effect of sediment strength, about which we do not have any lithological evidence. However, given our assumption that mechanical behavior depends on sediment composition, we can assume also implicitly that variations in sediment strength or composition may be incorporated into the model by changing the mechanical properties. The third case (EP1) corresponds to the study of sediment thickness influence. And the fourth case (CONV1) is related to the convergence rate contribution that is performed by changing the amount of horizontal loading acting on the model. The driving mechanism of the plates is carried out by transmission of the tangential pressure (driving by friction). Whatever the repartition of this pressure under the plate may be, it is by no means possible to know a priori the displacement field of the basal nodes resulting from this pressure. There is no chance a priori for this displacement field to be uniform. That is, 1500 bars does not represent the convergence rate of $2 \mathrm{~cm} / \mathrm{y}$. In particular, to prescribe to each of the basal nodes of the plate a displacement of a uniform amplitude and of variable ori-

Table 1. The mechanical properties used.

\begin{tabular}{ccccccccc}
\hline $\begin{array}{c}\text { Model } \\
\text { mechanical } \\
\text { units }\end{array}$ & $\begin{array}{c}E_{1} \\
\left(10^{6} \text { bar }\right)\end{array}$ & $\begin{array}{c}E_{2} \\
\left(10^{6} \text { bar }\right)\end{array}$ & $\begin{array}{c}G_{2} \\
\left(10^{6} \text { bar }\right)\end{array}$ & $\eta_{1}$ & $\eta^{2}$ & $\begin{array}{c}E_{\nu 1} \\
\left(10^{6} \text { bar }\right)\end{array}$ & $\begin{array}{c}E_{v 2} \\
\left(10^{6} \text { bar }\right)\end{array}$ & $\begin{array}{c}\eta \\
E_{1}\end{array}$ \\
\hline 1 & 0.8 & 1. & 0.3 & 0.3 & 0.3 & 0.8 & 1. & 0.8 \\
2 & 1.5 & 2. & 0.5 & 0.3 & 0.3 & 1.5 & 2. & 1.5 \\
3 & 0.02 & 0.05 & 0.01 & 0.4 & 0.3 & 0.02 & 0.05 & 0.02 \\
4 & 0.1 & 0.05 & 0.025 & 0.4 & 0.3 & 0.1 & 0.05 & 0.1 \\
& $0.1^{3}$ & $0.05^{\mathrm{a}}$ & 0.025 & 0.4 & 0.3 & 0.2 & 0.1 & 0.1 \\
& 0.2 & 0.1 & 0.025 & 0.4 & 0.3 & 0.1 & 0.05 & 0.2 \\
\hline
\end{tabular}

Note: Model mechanical unit 1 refers to characteristics related to the stratification plane [subducted plate $\left(\rho=2.7 \mathrm{~g} / \mathrm{cm}^{3}\right)$ ], whereas 2 refers to characteristics related to a direction perpendicular to the stratification plane loverriding plate $\left(\varphi=2.9 \mathrm{~g} / \mathrm{cm}^{3}\right)$ ); and $3=$ $\left.\mathrm{g} / \mathrm{cm}^{3}\right), \rho=$ density. $\mathrm{E}_{v 1}$ and $\mathrm{E}_{\nu,}$ are the Young's Modulus for the elasticity of the Kelvin $g / \mathrm{cm}$
Voigt elements. The last two lines of data represent values used for sediment strength models NA1 and NA2, respectively. entation in order to take the curvature of the plate into account should inevitably create artificial stresses inside the plate in the models. That is, it is convenient to prescribe a tangential pressure at the base (without any precise indication, this tangential pressure is assumed to be constant) so that the displacement field will freely adopt at the configuration that minimizes strain energy under this type of loading. The tangential pressure and the displacements of the basal nodes are in direct ratio. Thus keeping the total duration of flow constant variations in tangential pressure express variations in motion of the basal nodes.

\section{Boundary Conditions}

The left side (AE) of the model (Fig. 3) is held vertical, while the right side (CD) is subjected to passive loading induced by the weight of the surrounding rocks (geostatic loading). The underside of the subducted plate (BC), whose nodes are constrained to move along this boundary (BC), is subjected to a tangential pressure of 1500 bars in magnitude. The underside of the overriding plate $\left(\mathrm{AA}^{\prime}\right)$ slips freely on a horizontal plane (this adequately simulates the behavior of a very thick basement). The nodes of the truncated part of the subducted plate $\left(\mathrm{BB}^{\prime}\right)$ are constrained to move in the same direction as side $\mathrm{BC}$ at point $\mathrm{B}$. The top of the model is a free surface. These boundary conditions are the same in all models.

From finite element grids, mechanical assumptions, and boundary conditions, we try to derive strains, fracturs, and folds induced at each point inside the model. In other words, the model is used to indicate the likely type of resulting deformation.

\section{Stress Distributions}

Stress distributions shown in Figures 4 through 6 indicate that almost the entire sedimentary cover for each case (of the four different cases presented earlier) is subjected to compressive stresses. The computed stresses are large in the vicinity of the junction of the plates, yielding the overall uplift of this area. The maximum compressive stress trajectories form arcs whose radii of curvature increase with distance from the junction. Far away from this area, the orientations of these stresses are almost horizontal. Stress patterns generally remain the same for all cases. Only their magnitudes differ from one case to another. 

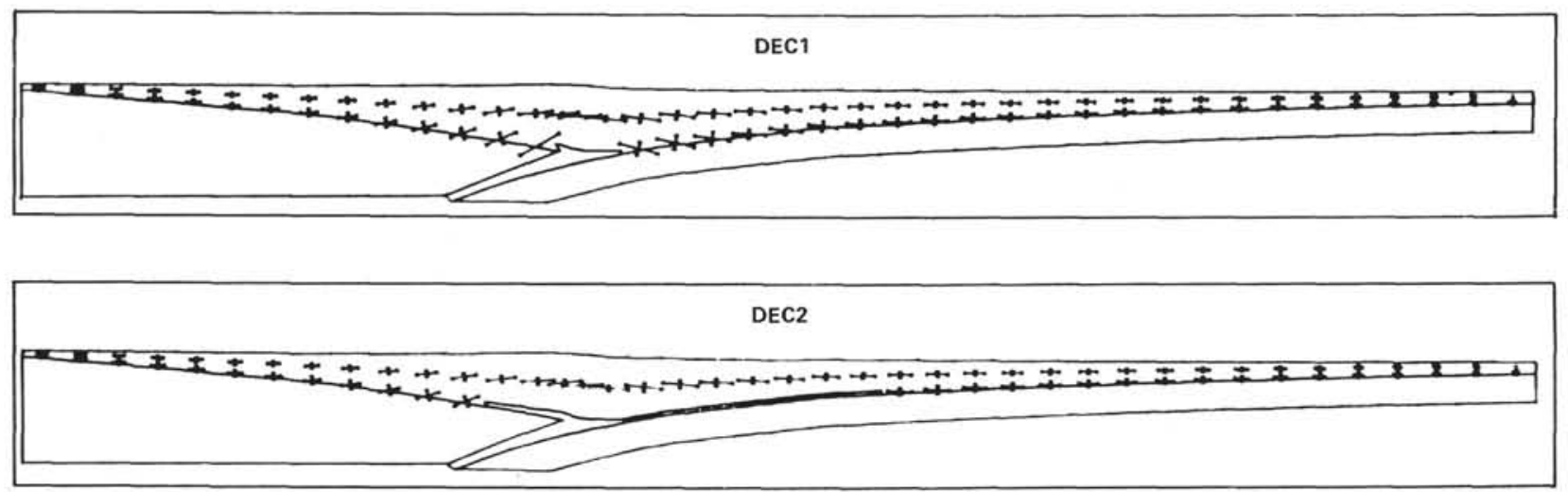

Stress distribution

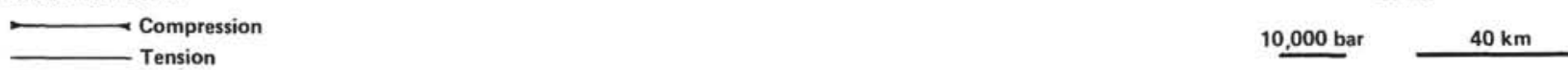

Figure 4. Case 1. Stress distribution of Models DEC1 and DEC2. (Model DEC1 preserves a strong coupling between units, whereas Model $\mathrm{DEC} 2$ has a thin weak transitional layer between the subducted plate and sediment. Young's Moduli of instantaneous and differred elasticity are equal, which means instantaneous and differred deformations each contribute half the total deformation.)
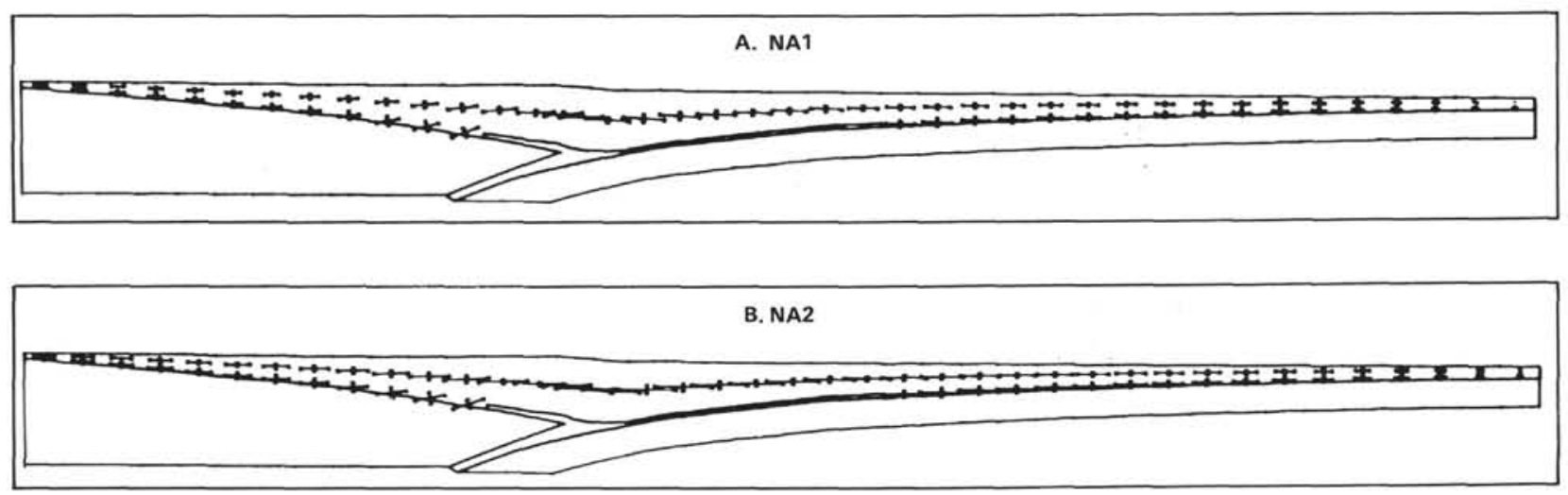

Stress distribution

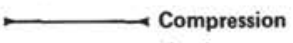

Tension

10,000 bar $40 \mathrm{~km}$

Figure 5. Case 2. Stress distribution of Models NA1 and NA2 (effect of changing mechanical properties and thus of sediment composition). A. Young's Modulus of differred elasticity is two times greater than that of instantaneous elasticity. Thus sedimentary cover undergoes more elastic deformation. B. Young's Modulus of instantaneous elasticity is two times greater than that of differred elasticity. Thus sedimentary cover displays more viscous deformation. Stresses here are large with respect to A.

\section{Possible Effects of Stresses on the Sedimentary Cover}

At each point of the model, we interpret the stresses and represent their possible effects on rocks. In other words, we do not assert that a given deformation will be observed at a given point. But we consider that if something appears at a given point, it could be a deformation of the type indicated. The failure criteria used take into account mean stress and deviatoric stress. If there is any tensile stress, the rock fails. When a mean stress is higher than a given limit (increasing ductility of rock) and the deviatoric stress is not too high in relation to mean stress, this stress state characterizes pseudoplasticity and suggests the possibility of rock-folding rather than fracturing. When the deviatoric stress exceeds a given limit, shear failure along conjuguate planes occurs. As the evaluated stresses do not have physical meaning (see earlier discussion) and have significance only with respect to the others, it is not possible to compare them with an experimental failure criterion directly. Comparisons also have to be made in a relative way-to determine which of two points A and B has the higher chance to fail or, in viscoelasticity rheology, which will fail first.

Usually the interpretation of the stresses computed by modeling consists of examining the orientation of the principal stresses point by point and in drawing from them, when the failure criterion is reached, the orientation of the corresponding fracture. Given these conditions and in the scale of our model, a great fault will be considered to be a great fracture propagating in a continuous medium and guided by the direction of maximum stress, which determines the trajectory of this fracture. Experiments on the decay of engineering or mining works have shown that the greatest discontinuities 

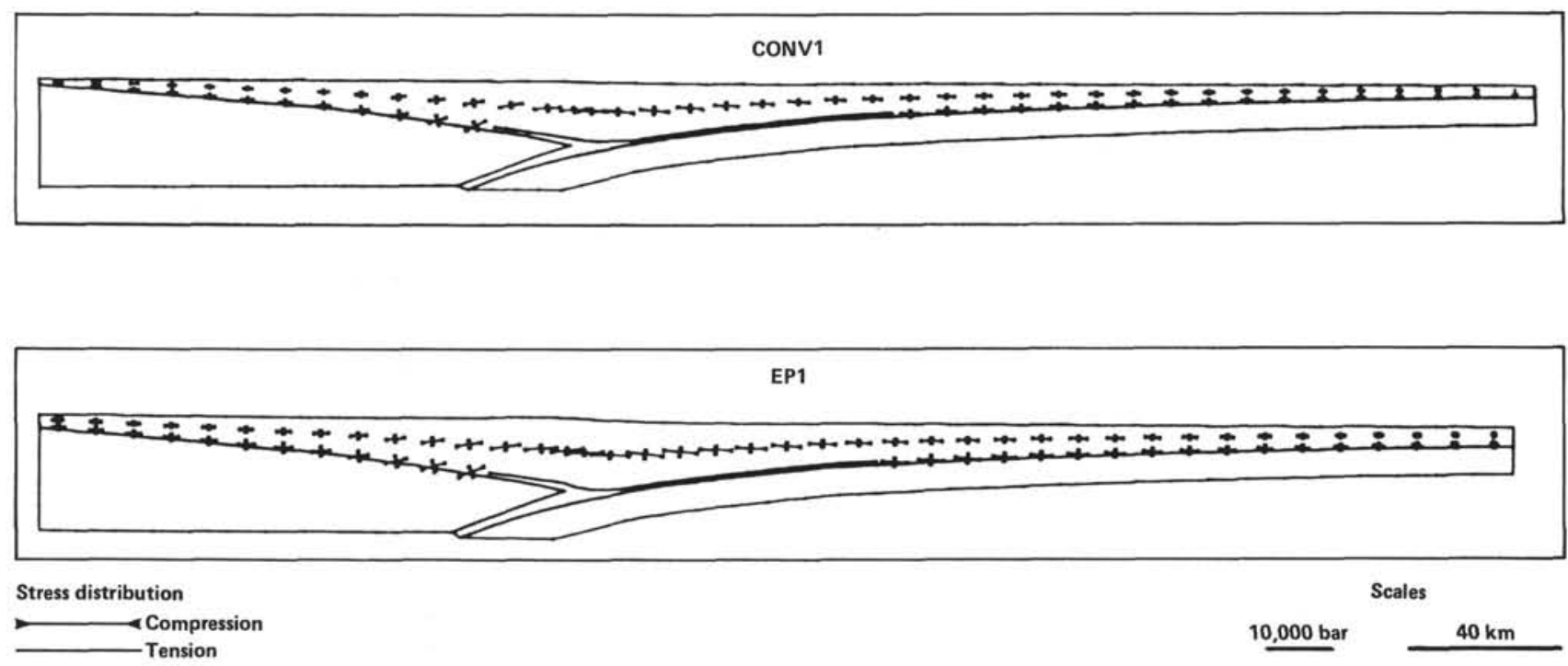

Figure 6. Cases 3 and 4. Stress distribution of Models CONV1 and EP1. In Case 3 (CONV1), the tangential pressure applied is reduced to 1000 bars. In Case 4 (EP1), $1000 \mathrm{~m}$ are added to sedimentary cover thickness of Model DEC2. Stresses here are almost equal to stresses of DEC2.

often appear along the boundaries between failed and unfailed zones, in contradiction to the direction of maximum stress. It is convenient, without being systematic or categorical, to examine seriously (if that is possible) the hypothesis that the formation of discontinuities occurs along the separations between zones that have been differentiated mechanically after a first phase of failure. In other words, we propose here to pay attention not only to the orientation of the stresses, but to the geometry of the boundaries between zones showing the greatest contrast in the degree of local failure.

If, following such interpretation, we have the configuration shown in Figure 7B, it should be possible to find the configuration shown in Figure 7C with another value of $k$. Whatever the real physical thresholds of failure may be, producing faulting or governing fault stabilization or amplification (keep in mind that there are several thresholds), the faults will have the patterns shown in Figure 7D. Thus it is more interesting to question the nature of faulting, and to observe whether the discontinuities having the patterns indicated exist in reality. We think that it is important not to discount this possibility before considering it carefully.

This is the idea, new in geology, but well-known in mining, that we wish to develop here. Thus Figure 8A-C represents what could happen with the assumptions we have made, inside the sedimentary cover at a converging plate boundary. The overall styles of deformation are similar in each case and occur in a zone adjacent to the plate junction, whereas their magnitudes change from one case to another.

\section{Parameter Influences}

The figures just described are concerned with the final deformation state. Other states are described elsewhere (Ngokwey, 1983).

Vertical displacements (v) versus time for the free surface at the vertical junction (see Fig. 3) are shown in
Figure 9. This figures provides some information about how events evolved with respect to time, allowing the influence of a given parameter to be shown more clearly. Despite constant boundary conditions for all the models, the curves representing vertical displacements versus time are quite different from one case to another, thus showing the influence of each parameter.

A comparison of Model DEC1, which maintains a strong coupling between units, with Model DEC2, which has a thin weak transitional layer, indicates that the existence of this particular layer decreases the amplitude of vertical displacements, and the distance between F1 and F3 increases. Strain rates are still equal. Assuming that tangential pressure reproduces the rate at which the basal nodes of the subducted plate are moving, we can see that if the tangential pressure acting on the model is low (1 kilobar or 1000 bars for Model CONV1), then the vertical displacement are lessened (the curve related to CONV1 is the lowest one). Curves representing the effects of differences in mechanical properties (thus in the nature of the sedimentary cover) show major differences. It can be seen that during the first half of the deformation time, the curves relating to models DEC2, NA1, and NA2 have very different shapes, yielding diverse strain rates. Let $\dot{\epsilon}_{y i}$ be the strain rates. During time intervals $\mathrm{T} 1, \mathrm{~T} 2, \mathrm{~T} 3$, and $\mathrm{T} 4, \dot{\epsilon}_{y i}$ varies in a significant way. During

$$
\begin{aligned}
& \text { T1: } \dot{\epsilon}_{y N A 1}<\dot{\epsilon}_{y D E C 2}<\dot{\epsilon}_{y N A 2} ; \\
& \text { T2: } \dot{\epsilon}_{y N A 1}<\dot{\epsilon}_{y D E C 2} \equiv \dot{\epsilon}_{y N A 2} ; \\
& \text { T3: } \dot{\epsilon}_{y N A 1} \equiv \dot{\epsilon}_{y N A 2}<\dot{\epsilon}_{y D E C 2} ; \\
& \text { T4: } \dot{\epsilon}_{y N A 1} \equiv \dot{\epsilon}_{y D E C 2}>\dot{\epsilon}_{y N A 2}
\end{aligned}
$$

The strong coupling model (DEC1) and the slow convergence model (CONV1) show respectively high and low strain rates. Note in Figure $8 \mathrm{C}$ that the existence of very thick sedimentary cover (Model EP1, where $1000 \mathrm{~m}$ were added to sediments of Model DEC2, as indicated in Fig. 6) provides possible higher-angle thrust faulting than Model DEC2. 
A

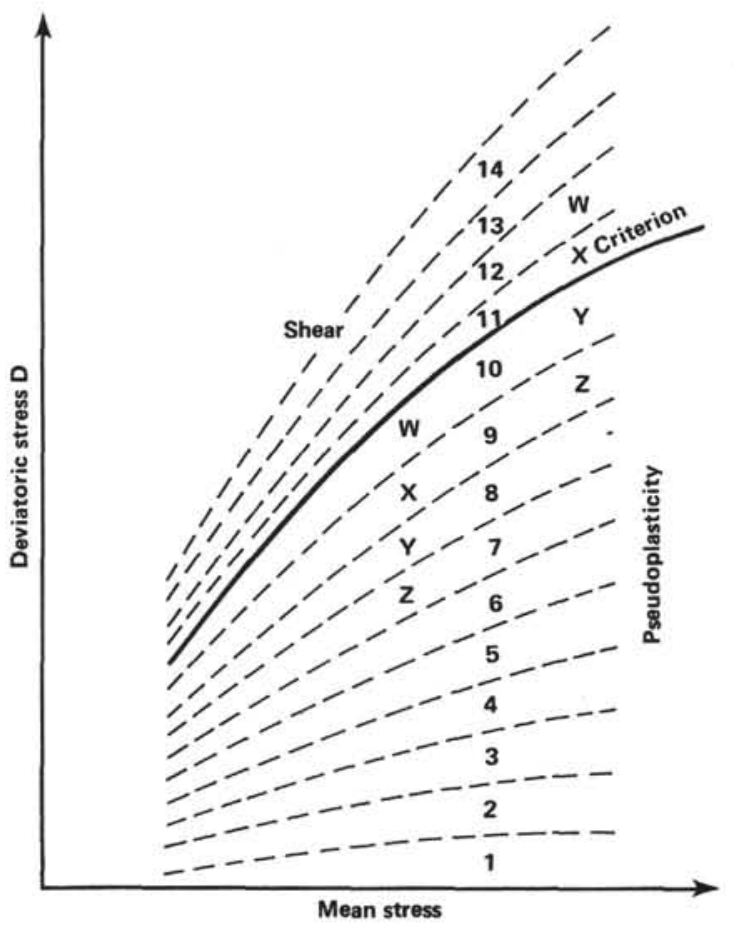

B

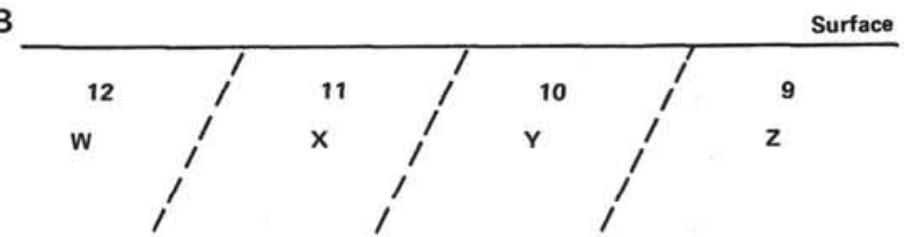

C

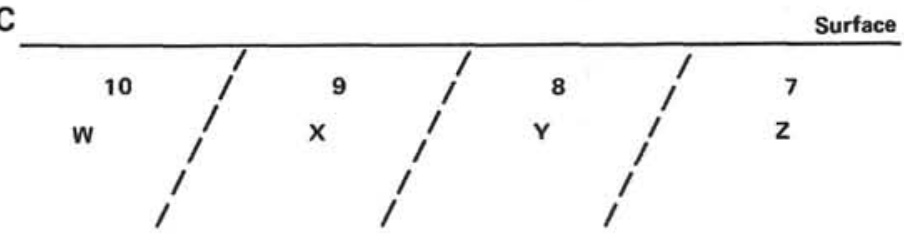

D

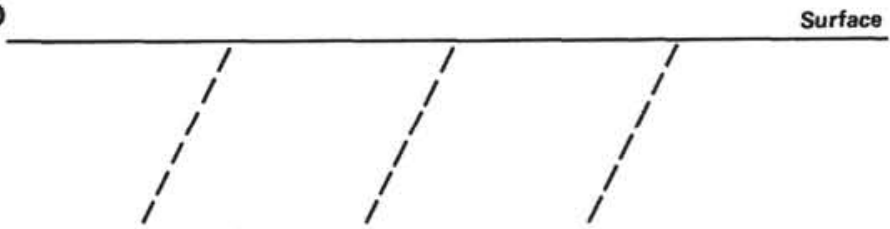

Figure 7. A-D. Relative risk of failure-theoretical example. The positions of the representative points (W-Z) of the computed stresses are characterized by the rank (i.e., 1-14) of the zones in which they are. In all cases, FR (failure risk) $z F_{y}<F_{x}<F_{w}$.
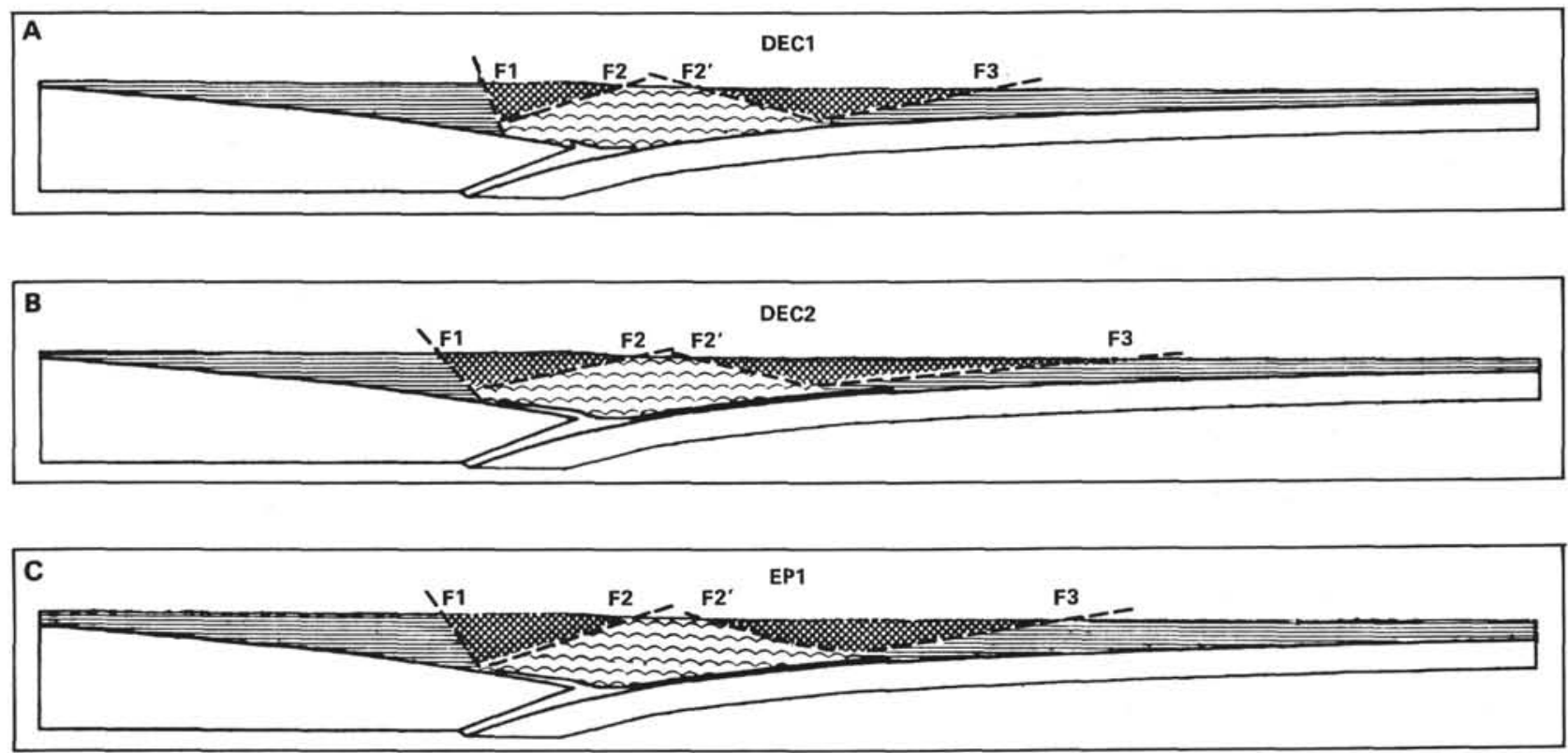

Legend

High failure risk zone

लm Pseudoplastic zone (folding)

$40 \mathrm{~km}$

Low failure risk zone

Figure 8. Possible effects of stress distribution of Models DEC1 (A), DEC2 (B), and EP1 (C). These drawings represent the possible deformation types that could be observed in sediment. Notice the dips of possible fracture F3. 


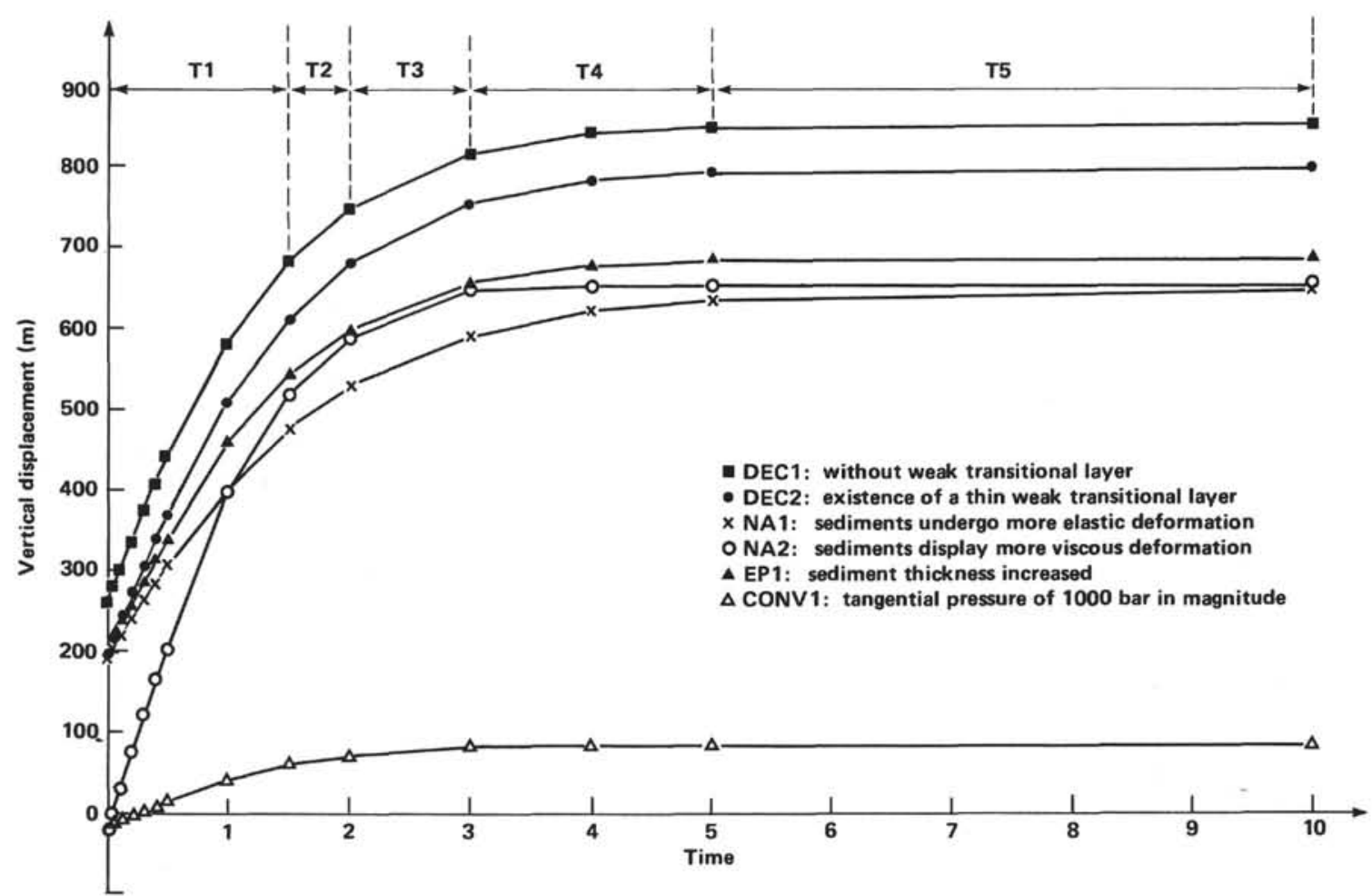

Figure 9. Vertical displacements versus time for the free surface at the vertical junction of the converging plates (see Fig. 3). (The underside of Models DEC1, DEC2, NA1, NA2, and EP1 was subjected to a tangential pressure of 1500 bar in magnitude.)

In summary, the existence of a thin weak transitional layer and the mechanical properties of the sedimentary cover very strongly influence the models, while convergence rates and sediment thickness affect only the amplitude of deformation.

\section{COMPARISON WITH BARBADOS RIDGE COMPLEX}

The results of these elementary models form a basis for discussion of observations made in the case of the Lesser Antilles island arc. This area was selected for the comparison for two reasons: first, because the geodynamic context is relatively simple, and second, because of the occurrence of very thick sedimentary accumulations in its southern part where the geometry of structures is discernable by seismic reflection techniques. We now test the compatibility of the models with the structural features encountered in this area.

\section{Tectonic Styles}

Let us examine Figure 10, in which we compare observations of the Barbados Ridge complex (after Westbrook, 1982) with the results obtained from Model DEC2 (having a weak transitional layer that is analogous to a décollement).

\section{East-West Compressional Stress Field and Low-Angle Thrusts}

The calculations described earlier indicate that the model permits the calculation of the compressional stress field.
Fitch and Scholz (1971) stated that compressive stresses give rise to low-angle thrust faults in subduction zones. From our model, the existence of a thin weak transitional layer provides a stress field leading to low-angle thrust faulting (Fig. 10). The dip of the possible fracture F3 in the model having a weak transitional layer (DEC2) is higher than the dip of the same fracture in the strong coupling model (DEC1). Close to the junction of the plates, stresses are large enough to yield large vertical displacements of the free surface. The junction between the North American and Caribbean plates is commonly defined by means of the negative Bouguer gravity anomaly axis crossing over Barbados Island. Thus the model agrees with the assumption that Barbados Island rises at the vertical junction of the plates as a consequence of subduction (Westbrook, 1975).

East of Barbados Island, overthrusts have eastward or seaward vergence, whereas overthrusts with landward or westward vergence occur to the west of Barbados. Our model provides these phenomena too (fractures F1 and F3). Nevertheless, we cannot give a satisfactory explanation for possible fracture $\mathrm{F} 2{ }^{\prime}$. It does not fit well the observations made in the Barbados Ridge complex. Notice that possible fractures F1, F2, F2', and F3 have been drawn according to the procedure discussed earlier in the section on possible effects of stresses on the sedimentary cover.)

\section{Shortening}

At converging margins, lithospheric plates shorten, as do sediments (Seely et al., 1974; Mascle et al., in 

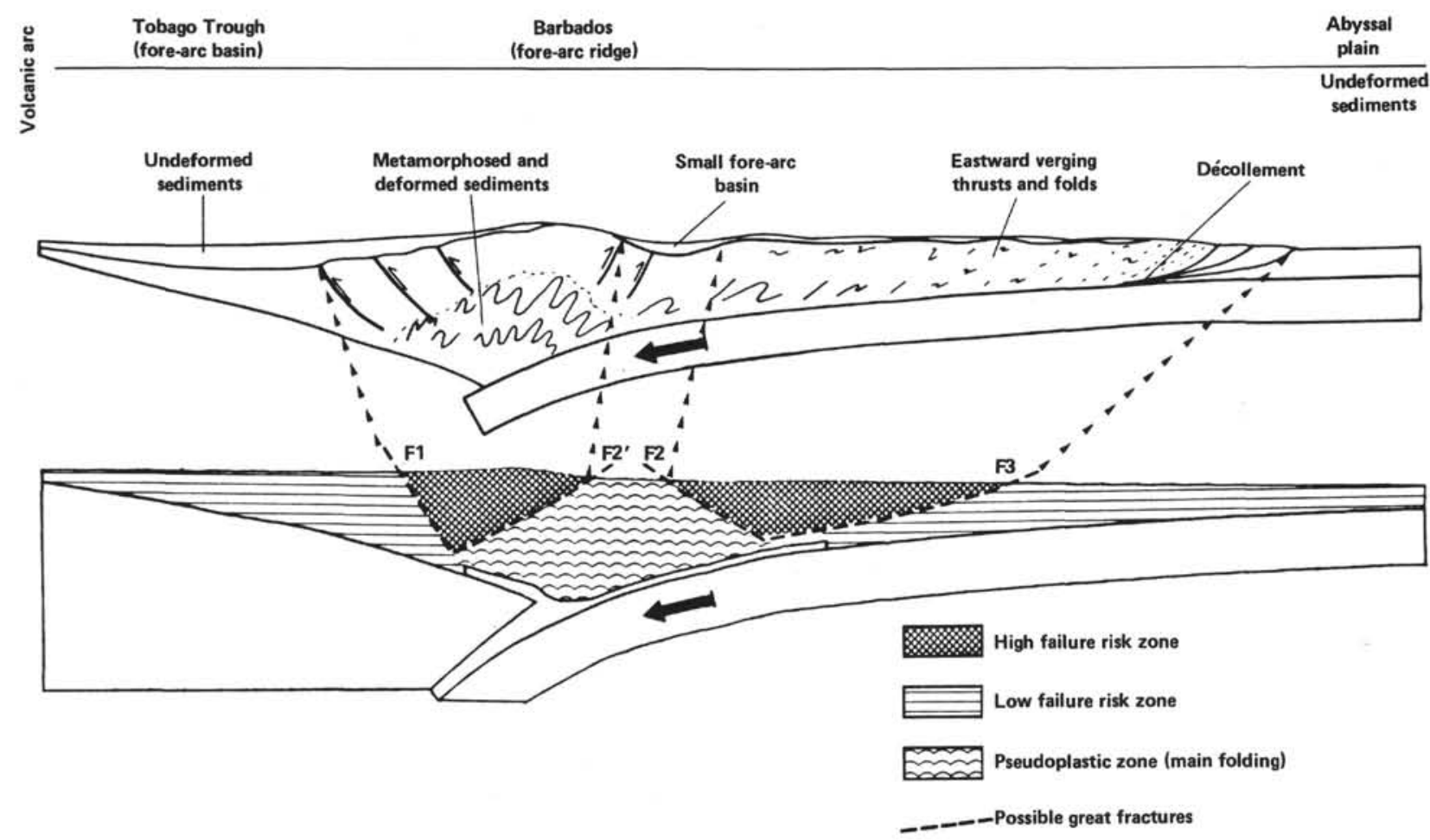

Figure 10. Comparison of Model DEC2 (see Figs. 4 and 8) with the Barbados Ridge complex. The diagrammatic cross section is from Westbrook (1982). (See text for discussion.)

press). Shortening is an integral part of our model. In the vicinity of the plate junction, shortening is much less than in areas far from the junction. Shortening is typically greatest close to the area where the weight of overburden is not great enough to impede it. This factor leads us to assume that the shortening at the deformation front of the Barbados Ridge complex may now be greater than in the area close to Barbados Island itself.

In summary, the results of the models presented qualitatively fit observations of the Barbados Ridge complex in the following ways:

1. Sediment deformation appears to occur preferentially in a zone adjacent to the plate junction and is bounded by fractures or faults.

2. The tectonic style is characterized mainly by lowangle thrust faulting along the décollement (thin weak transitional layer).

3. The area corresponding to the Tobago Trough and abyssal plain display a remarkable lack of large deformations.

4. Both frontal eastward and back westward verging overthrusts occur.

5. Barbados Island was formed by uplifting of sediments as a consequence of convergence at the junction of the North American and Caribbean plates.

\section{CONCLUSIONS}

The creation of any successful model implies knowledge of the pertinent data, but in this case many pertinent data remain unknown. The set of assumed mechanical units could have been affected by some inhomoge- neities (overpressured shales, differential compaction, geometric irregularities at the tops of both subducted plate and sedimentary cover, and so on) whose influence may have been appreciable during the process of geological deformation. It seems likely that these data will always remain beyond our reach. This being so, this study has been carried out with some hypotheses that are not rigorously exact but are nevertheless reasonable. Models were developed without taking into account the effects of isostatic response or temperature (providing thermal expansion), which could modify the stress field and thus deformation.

Despite these problems, a mathematical model for large deformations applied to the overthrusting of two lithospheric plates can be used to illustrate the deformation of the sediments and to analyze the influence of the different parameters as they are varied systematically.

The main results obtained in the present study are as follows:

1. Calculations indicate that a subduction model produces the compressional stress field inside sedimentary cover.

2. The distribution of the stress magnitudes plays an important role in the location of deformation types.

3. The existence of a thin weak transitional layer between units could have appreciable effects on the mechanism of low-angle thrust faulting.

4. Sediment composition provides more variations in strain rates and therefore in the ability of sedimentary cover to be deformed.

5. Sediment thickness and convergence rate influence the magnitude of the deformation. 


\section{ACKNOWLEDGMENTS}

I thank Jacques Quiblier for his useful discussions and advice. I am grateful to DR. Dan Davis and Dr. Graham K. Westbrook for critically reading the manuscript and providing many useful comments. Nevertheless I remain solely responsible for the ideas presented here as well as the form of the presentation.

\section{REFERENCES}

Fitch, T. J., and Scholz, C. H., 1971. Mechanism of underthrusting in Southwest Japan. A model of convergent plate interactions. $J$. Geophys. Res., 76:260-292.

Mandel, J., 1966. Cours de Mécanique des Milieux Continus: Paris (Gauthier-Villars).

Mascle, A., Biju-Duval, B., Letouzey, J., Montadert, L., and Ravenne, C., 1976. Sediments and their deformations in active margins off different geological settings. In International Symposium on Geodynamics in Southwest Pacific, Noumea, 1976. Paris (Technip Ed.) pp. 327-344.

Mascle, A., Lajat, D., and Nely, G., in press. Sediment deformation linked to subduction and to argillokinesis in the southern Barbados Ridge from multichannel seismic surveys. Trans. 4th Latin American Geol. Congr.
Ngokwey, K., 1983. Application de la méthode des eléments finis a l'étude des déformations des sédiments au front des marges actives-exemple de l'Arc Insulaire des Petites Antilles [Ph. D. dissert.]. Université Paris Sud-Orsay.

Oxburgh, E. R., and Turcotte, D. L., 1971. Origin of paired metamorphic belts and crustal dilation in island arc region. J. Geophys. Res., 76:1315-1327.

Pautot, G., 1975. Marges actives: Pérou et autres exemples. Bull. Soc. Geol. France, (7), 17:529-543.

Seely, D. R., Vail, P. R., and Walton, G. G., 1974. Trench-slope model. In (Burk, C. A., and Drake, C. L. (Eds), The Geology of Continental Margins: New York (Springer-Verlag), pp.249-261.

Westbrook, G. K., 1975. The structure of the crust and the upper mantle in the region of Barbados and the Lesser Antilles. Geophys. J. R. Astron. Soc., 43:201-242.

, 1982. The Barbados Ridge complex; tectonics of a mature forearc system. In Leggett, J. K. (Ed.), Trench-Forearc Geology, Geol. Soc. Spec. Publ. 10:275-290.

Date of Initial Receipt: October 19, 1982

Date of Acceptance: October 14, 1983 